\title{
Electronic structure and Fermi surface of Bi(100)
}

\author{
Ph. Hofmann, ${ }^{1, *}$ J. E. Gayone, ${ }^{2}$ G. Bihlmayer, ${ }^{3}$ Yu. M. Koroteev, ${ }^{4,5}$ and E. V. Chulkov ${ }^{4,6}$ \\ ${ }^{1}$ Institute for Storage Ring Facilities, University of Aarhus, DK-8000 Aarhus C, Denmark \\ ${ }^{2}$ Institute for Storage Ring Facilities, University of Aarhus, 8000 Aarhus C, Denmark \\ and Centro Atomico Bariloche and CONICET, 8400 S.C. de Bariloche, Argentina \\ ${ }^{3}$ Institut für Festkörperforschung, Forschungszentrum Jülich, D-52425 Jülich, Germany \\ ${ }^{4}$ Donostia International Physics Center (DIPC), 20018 San Sebastián, Basque Country, Spain \\ ${ }^{5}$ Institute of Strength Physics and Materials Science, Russian Academy of Sciences, 634021 Tomsk, Russia \\ ${ }^{6}$ Departamento de Física de Materiales and Centro Mixto CSIC-UPV/EHU, Facultad de Ciencias Químicas, UPV/EHU, \\ Apartado 1072, 20080 San Sebastián, Basque Country, Spain
}

(Received 5 October 2004; published 24 May 2005)

\begin{abstract}
The surface electronic structure of $\mathrm{Bi}(100)$ was studied by angle-resolved photoemission and the fullpotential linearized-augmented plane-wave film method. Experimentally, several electronic surface states were identified in the gaps of the projected-bulk band structure close to the Fermi level. Theory shows that these states belong to a spin-orbit split-surface band that extends through the whole Brillouin zone, and that some surface states penetrate very deeply into the bulk. In the experiment, the surface Fermi surface was found to consist of three features: an electron pocket at the $\bar{\Gamma}$ point, a hole pocket in the $\bar{\Gamma}-\bar{M}$ direction (i.e., in the direction of the surface-mirror line), and a small Fermi-surface element close to the $\bar{M}^{\prime}$ points.
\end{abstract}

DOI: 10.1103/PhysRevB.71.195413 PACS number(s): 79.60.Bm, 71.18. $+\mathrm{y}$, 73.20. $-\mathrm{r}, 79.60 .-\mathrm{i}$

\section{INTRODUCTION}

The group-V semimetal $\mathrm{Bi}$ has an interesting electronic structure. The Fermi surface fills only a very small fraction of the Brillouin zone (BZ). It consists of electron pockets around the $L$ points and hole pockets around the $T$ points of the BZ (see Fig. 2). The Fermi energy determined from the carrier density at these pockets is very small, around $30 \mathrm{meV}$, and the effective masses of the carriers is also small in certain directions. ${ }^{1,2}$ This peculiar electronic structure is closely related to the rhombohedral $(A-7)$ geometric structure. For $\mathrm{Bi}$ in other configurations than the bulk $A-7$ crystal, very different properties can be expected for two reasons. First, the change in the geometric structure could turn the system either into a better metal or into a semiconductor. Second, the long, effective de Broglie wavelength of the bulk carriers combined with the long mean-free path leads to pronounced quantum-size effects for systems with a bulklike structure, but a finite size. Such interesting changes of the electronic structure have indeed been predicted and observed for thin films, ${ }^{3-5}$ clusters, ${ }^{6}$ and nanotubes. ${ }^{7-9}$

Other cases in which structural changes can be expected to lead to interesting consequences for the electronic structure are the surfaces of $\mathrm{Bi}$. For the surface orientations studied so far, (110) and (111), the surface is a much better metal than the bulk due to the presence of electronic surface states. ${ }^{10-12}$ In this paper, we discuss the electronic structure of the $\mathrm{Bi}(100)$ surface, which we also find to be a better metal than the bulk. In contrast to the (110) and (111) surfaces, however, the surface states on $\mathrm{Bi}(100)$ penetrate very deeply into the bulk. We suggest that this could help to explain some apparent contradictions found for the electronic structures of clusters and nanotubes.

At this point we add a brief comment about the crystallographic notation of the bismuth surfaces and their relations to the bulk structure. In bulk $\mathrm{Bi}$, each atom has three equidistant nearest-neighbor atoms and three equidistant nextnearest neighbors slightly further away. This results in puckered bilayers of atoms perpendicular to the (111) direction, in which each atom is covalently bonded to its three nearest neighbors. Each atom's next-nearest neighbors are in the adjacent bilayers, and the bonding within each bilayer is much stronger than the interbilayer bonding. This explains why $\mathrm{Bi}$ crystals easily cleave in the (111) plane. The rhombohedral A-7 structure has two atoms per bulk unit cell, corresponding to the two atoms in the bilayers. Apart from being rhombohedral, the $A-7$ structure is also hexagonal with six atoms per unit cell, and in the literature both rhombohedral and hexagonal indices can be found. To confuse matters even more, one also finds indices referring to the pseudocubic nature of the structure and even a hexagonal notation in which one of the four (redundant) indices is omitted. The relation between these notations is described in the work of Jona ${ }^{13}$ and in considerable detail, also including the (100) surface, in Ref. 14. In the present paper only the rhombohedral notation is used unless stated otherwise. Our (100) surface thus corresponds to a pseudocubic (111) and a hexagonal (0111) surface. The close-packed (111) surface is also a pseudocubic (111) surface and a hexagonal (0001) surface.

Before giving our results we describe the structure of $\mathrm{Bi}(100)$ both in real and reciprocal spaces and compare it to that of $\mathrm{Bi}(111)$. Figure 1 shows the geometry of $\mathrm{Bi}(100)$. The structure can be viewed as built from quasihexagonal layers. In each layer an atom has two neighbors at the same distance as on the $\mathrm{Bi}(111)$ surface $(4.54 \AA)$ and four next-nearest neighbors at a slightly larger distance (4.72 $\AA$ ). The three nearest neighbors of any $\mathrm{Bi}$ atom in the bulk structure, however, do not lie in the same quasihexagonal layer, but they connect these layers. The connections to the nearest neighbors have been indicated as "bonds" in the figure. The side 


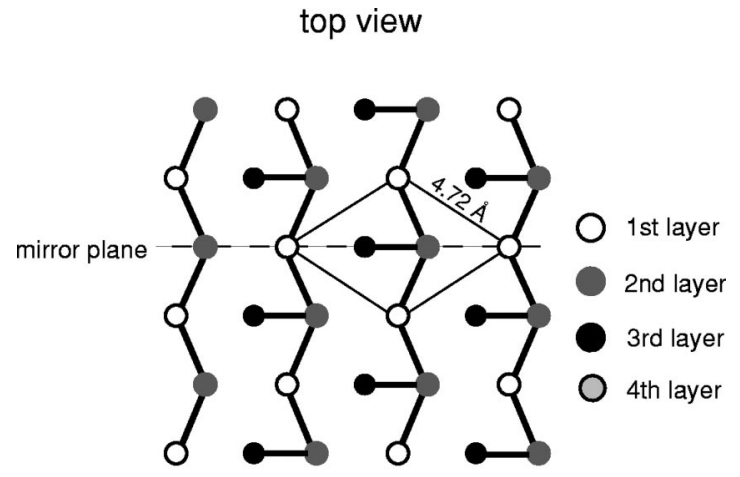

side view (parallel to mirror plane)

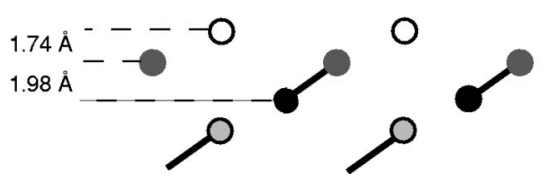

FIG. 1. Truncated bulk structure of $\mathrm{Bi}(100)$ (termination with a short interlayer spacing). In the top view the surface unit cell is shown. The dashed line is the mirror plane of $\mathrm{Bi}$, the only symmetry element on this surface. The nearest-neighbor atoms are connected by lines. The side view shows a quasi- $A B C A B C \cdots$ stacking sequence. However, the fourth layer is shifted by $0.57 \AA$ along the mirror plane with respect to the first layer. Only the bonds in the paper plane are indicated in the side view.

view shows that there are two different possible interlayer spacings. The termination of the surface is not known but it seems likely that the shorter interlayer spacing prevails, not the least because it requires the breaking of only one covalent bond per unit cell instead of two. It can be seen that the structure is close to an $A B C A B C \cdots$ stacking sequence of the quasihexagonal layers, but not quite; the fourth-layer atoms are only almost in registry with the first-layer atoms. They are actually shifted by an amount of $0.57 \AA$. The symmetry of the surface is very low. The only symmetry element is a mirror plane, which is also indicated in the figure. There are thus some important differences between $\mathrm{Bi}(100)$ and $\mathrm{Bi}(111)$, even though both are pseudocubic (111) surfaces; in order to form $\mathrm{Bi}(111)$ no covalent next-neighbor bonds have to be broken, while one bond per unit cell has to be broken to form $\mathrm{Bi}(100)$. $\mathrm{Bi}(111)$ also possesses a higher symmetry with a threefold axis and three mirror planes.

The top part of Fig. 2 shows the Brillouin zone of Bi and a sketch of the bulk Fermi surface (not to scale). There are two types of well-separated Fermi surface elements: hole pockets at the $T$ points and electron pockets at the $L$ points. Also shown is the projection of the surface Brillouin zone (SBZ) of Bi(100). The bottom of Fig. 2 shows the SBZ with the symmetry points. It actually shows an extended zone scheme that we will need when discussing the experimental results. The indices at the symmetry points will also be used for this purpose. The points at the corners of the SBZ and the middle points of the sides have been called $\bar{K}$ and $\bar{M}$, respectively. This notation emphasizes the similarity to a hexagonal surface. Note, however, that there are two different types of $\bar{M}$ points: the points we call $\bar{M}^{\prime}$ and the ones we call simply
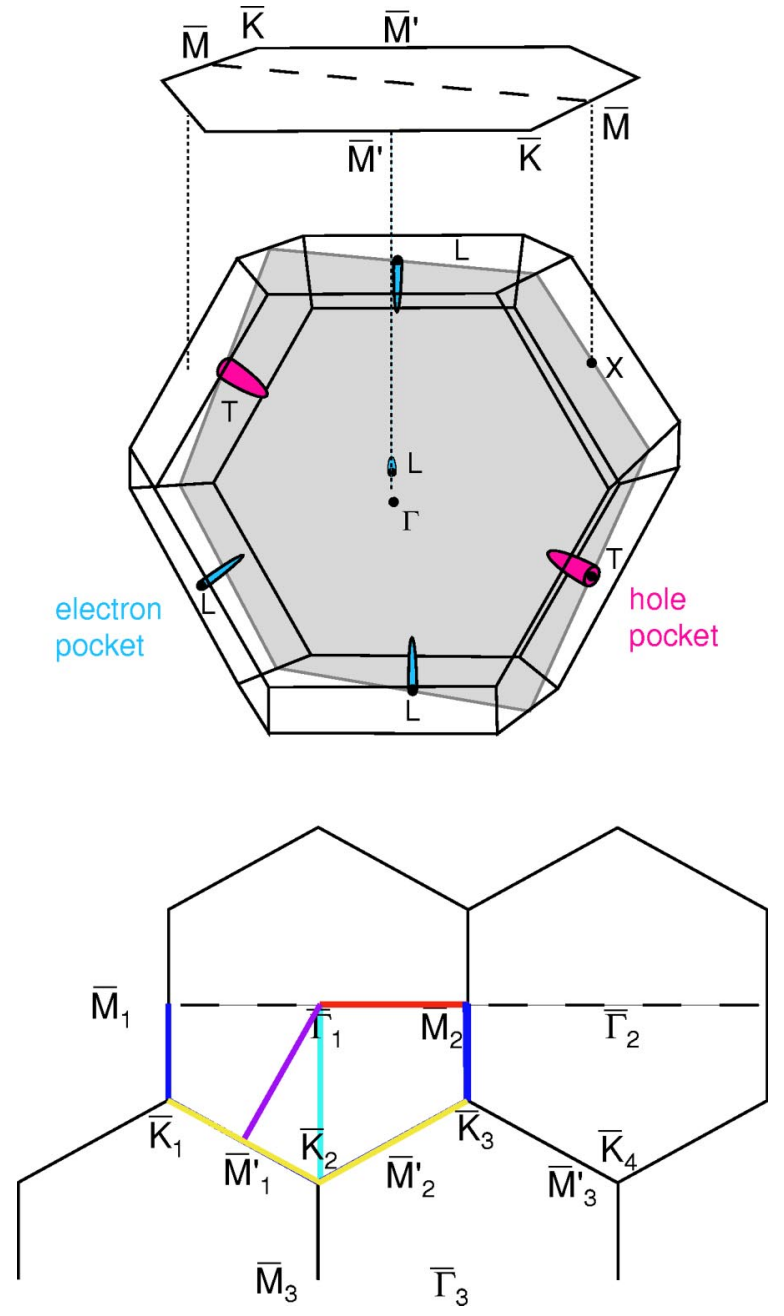

FIG. 2. (Color online) Top: Bulk Brillouin zone of Bi and projection onto the (100) surface. The elements of the bulk Fermi surface are indicated, but not to scale. The shaded plane is the bulkmirror plane, and the dashed line is its projection onto the surface. Bottom: The surface Brillouin zone of $\mathrm{Bi}(100)$ in an extended-zone scheme. The dashed line is the mirror line. The colored lines in the bottom part of the first surface Brillouin zone correspond to the lines along which the electronic dispersion was measured and calculated (see figures below).

$\bar{M}$. As for the real-space geometry, the only relevant symmetry element for this surface is a bulk-mirror plane that is projected onto a surface-mirror line. Both are shown in the figure.

Another symmetry which is important for the electronic states of low-symmetry surfaces such as $\mathrm{Bi}(100)$ is timereversal symmetry. For nonmagnetic systems, this symmetry leads to the relation

$$
E\left(\vec{k}_{\|}, \uparrow\right)=E\left(-\vec{k}_{\|}, \downarrow\right),
$$

which means that if one has a surface state at $\vec{k}_{\|}$with a binding energy $E$ and a spin $\uparrow$, then there must also be a state at $-\vec{k}_{\|}$with the same energy but spin $\downarrow$. Consider for example the $\bar{M}_{1}$ point in Fig. 2 . Time-reversal symmetry causes the 
$\bar{M}_{2}$ point to have energetically degenerate electronic states, but with opposite spin. More generally, the combination of time-reversal and mirror symmetry adds considerably to the expected symmetry in the dispersion of the electronic states. In particular it causes the dispersion in the "left" and the "right" halves of the SBZ to be the same, as if there were a second-mirror line perpendicular to the real-mirror line. This is of course only true in a non-spin-resolved experiment.

\section{METHODS}

The $\operatorname{Bi}(100)$ crystal was mechanically polished and cleaned in situ by cycles of $\mathrm{Ar}^{+}$and $\mathrm{Ne}^{+}$sputtering and annealing to $200^{\circ} \mathrm{C}$. The surface cleanliness was monitored with Auger electron spectroscopy (AES) and photoemission. In the initial stages of cleaning a clear oxygen peak was found in the valence band. Later, the surface quality was judged by the shape and intensity of the surface states. No contaminations could be observed by AES in the latter stages of cleaning. The surface was found to stay free of detectable contaminations for a very long time. The line shape of the surface states was not found to change within about $24 \mathrm{~h}$ after cleaning, and the surface-state peaks were still observable after several weeks in a vacuum without cleaning. The surface order was checked by low-energy electron diffraction (LEED). Our $\mathrm{Bi}(100)$ crystal showed a fair-quality $(1 \times 1)$ LEED pattern.

The photoemission experiment was performed using an angle-resolved electron spectrometer at the SGM-3 beamline on the undulator of the storage ring ASTRID in Aarhus. A detailed description of the instrument is given elsewhere. ${ }^{15}$ In short, the beamline covers an energy range from $12 \mathrm{eV}$ to $140 \mathrm{eV}$, with a resolving power better than 15000 . For the measurements reported here, the light was incident under an angle of $50^{\circ}$ away from the surface normal with the polarization vector of the light coinciding with the mirror plane of the crystal. The electron spectrometer was a commercial VG ARUPS 10 analyzer that was mounted on a goniometer inside the chamber and equipped with a multichannel detector. The analyzer position could be changed by motors outside the chamber. The total energy resolution used in this work was $30 \mathrm{meV}$. The angular resolution was around $\pm 0.7^{\circ}$. The sample temperature was around $30 \mathrm{~K}$. The pressure during the experiments was in the mid- $10^{-11}$-mbar range.

We also performed $a b$ initio calculations of the surface electronic structure of $\mathrm{Bi}(100)$. The full-potential linearized augmented plane-wave method in film geometry, ${ }^{16,17}$ as implemented in the FLEUR code was used and the local density approximation ${ }^{18}$ to the density functional theory was employed. Spin-orbit coupling was included in the selfconsistent calculations. ${ }^{19}$ To simulate the surface, we used a 22-layer $\mathrm{Bi}(100)$ film terminated with $\mathrm{H}$ on one side of the film. The hydrogen partially saturated the broken Bi bonds at the surface and helped to minimize the interaction between the surface states of the two surfaces of the film. The geometry was chosen such that the first interlayer distance $\left(d_{12}\right)$ on each side of the film was the shorter one of the two possible interlayer distances. The atoms in the first four layers on the

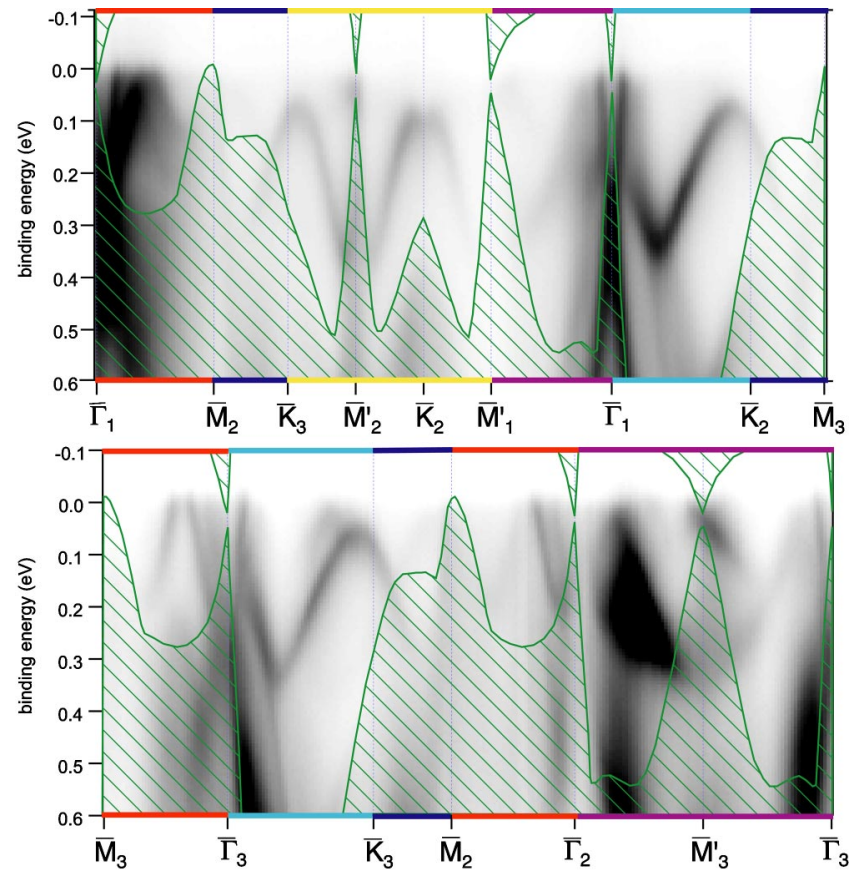

FIG. 3. (Color online) Measured dispersion of the electronic states along the several directions in the SBZ, taken at $h \nu=19 \mathrm{eV}$. The photoemission intensity is plotted as a function of binding energy and $\vec{k}_{\|}$. The dark correspond to high intensity. The green hashed area is a projection of the calculated tight-binding band structure after Ref. 20. The colored horizontal bars can be used to identify equivalent directions in the extended-zone scheme.

clean side (i.e., without $\mathrm{H}$ ) of the film were allowed to relax. We found a contraction of $d_{12}$ by $3 \%$, followed by a large (14\%) expansion of $d_{23}$ and a contraction of the third interlayer distance.

\section{RESULTS AND DISCUSSION}

Figure 3 shows the measured dispersion of the electronic states along the several directions in the extended zone scheme. The equivalent directions are marked with identically colored bars for easier comparison. The individual spectra were taken in steps of constant $\vec{k}_{\|}$of approximately $0.02 \AA^{-1}$. Around normal emission this corresponds to an angular step of $0.6^{\circ}$. The figure shows a gray-scale plot of the photoemission intensity as a function of binding energy and $\vec{k}_{\|}$. The intensity is saturated at some points, the black areas, in order to make the less-intense features also visible. The values of $\vec{k}_{\|}$were calculated using a binding energy of $0 \mathrm{eV}$, which means that the scale is exactly correct only at the Fermi level. However, since the whole binding-energy range is only $600 \mathrm{meV}$, the error induced by this is very small. Apart from the photoemission data, the figure also shows the result of a band-structure projection on this surface, which was calculated using the tight-binding scheme of Liu and Allen. ${ }^{20}$ Extended gaps in the projected bulk-band structure around the Fermi level can be found, as expected for a semimetal.

Surface states can easily be identified by three criteria. First, they appear in the projected bulk band gaps. Second, 
they are also observable at other photon energies, and third, the same states can be observed at symmetry-equivalent $\vec{k}_{\|}$ points in the extended zone scheme, albeit with very different intensities. Using these criteria, we can identify several surface states, e.g., three very steep bands close to $\bar{\Gamma}$ in the $\bar{\Gamma}_{1}-\bar{M}$ direction and one in the $\bar{\Gamma}-\bar{K}$ direction.

The symmetry of $\mathrm{Bi}(100)$ that we discussed above is reflected in the measured dispersions. Consider for example the $\bar{K}_{2}$ and $\bar{K}_{3}$ points that are related by the combination of mirror and translational symmetry. For both a clear surfacestate peak is found at a binding energy around $80 \mathrm{meV}$. The same applies for the three $\bar{M}^{\prime}$ points where a surface state can be found at, or very close to, the Fermi level. Another example is the dispersion in the $\bar{\Gamma}_{1}-\bar{K}_{2}$ direction that is also observed in the $\bar{\Gamma}_{3}-\bar{K}_{3}$ direction. Furthermore, it is clearly seen that the $\bar{M}^{\prime}$ and $\bar{M}$ points are inequivalent.

A closer inspection of the band dispersion shows that the extrema are often not located at the points on the SBZ boundary. One example is the dispersion along $\bar{\Gamma}_{1}-\bar{K}_{2}$ and $\bar{\Gamma}_{3}-\bar{K}_{3}$ that was discussed above. This effect leads also to a peculiar dispersion in the $\bar{M}_{2}^{\prime}-\bar{K}_{2}-\bar{M}_{1}^{\prime}$ direction where a small "dip" in the dispersion can be observed at $\bar{K}_{2}$. The actual binding-energy minimum is not found at $\bar{K}$, but close to this point. It has to be pointed out that there is no symmetry requirement that would force the state to have the lowest binding energy exactly at the $\bar{K}$ points.

There are some details for which the situation remains somewhat unclear. One is the small shoulder which develops in the $\bar{\Gamma}_{3}-\bar{K}_{3}$ direction close to the Fermi level. This feature cannot be observed along $\bar{\Gamma}_{1}-\bar{K}_{2}$. A possible reason for this could be that its intensity in the latter direction is simply too small. Another case of an unclear dispersion is found in the $\bar{\Gamma}_{2}-\bar{M}_{3}^{\prime}$ direction, where an intense and apparently very broad feature is observed. This, however, is an artifact of the way the data are presented in Fig. 3; the intensity along this direction is so high that the gray scale is saturated. The actual dispersion of the surface state in the $\bar{\Gamma}_{2}-\bar{M}_{3}^{\prime}$ is very similar to that in the equivalent directions.

The advantage of measuring the dispersion along more than one symmetry-equivalent direction has already become apparent. It turns out to be crucial when we now direct our attention to the Fermi-level crossing of the surface states, the most interesting subject in the study of a semimetal surface. As we have discussed above, a very clear Fermi-level crossing is seen close to $\bar{\Gamma}_{1}$ along $\bar{\Gamma}_{1}-\bar{K}_{2}$. A crossing from the same state can also be seen when going from $\bar{\Gamma}_{1}$ towards $\bar{M}_{2}$. However, when continuing in this direction the situation is somewhat unclear. The background intensity is high everywhere around normal emission, and it is hard to determine the individual bands. Much more revealing is the symmetryequivalent $\bar{\Gamma}_{3}-\bar{M}_{3}$ direction. The Fermi-level crossing close to $\bar{\Gamma}_{3}$ is weak but visible, and continuing towards $\bar{M}_{3}$ one clearly observes two more crossings. The positions of the crossings can be seen more quantitatively in Fig. 4, where we have plotted the photoemission intensity integrated over a

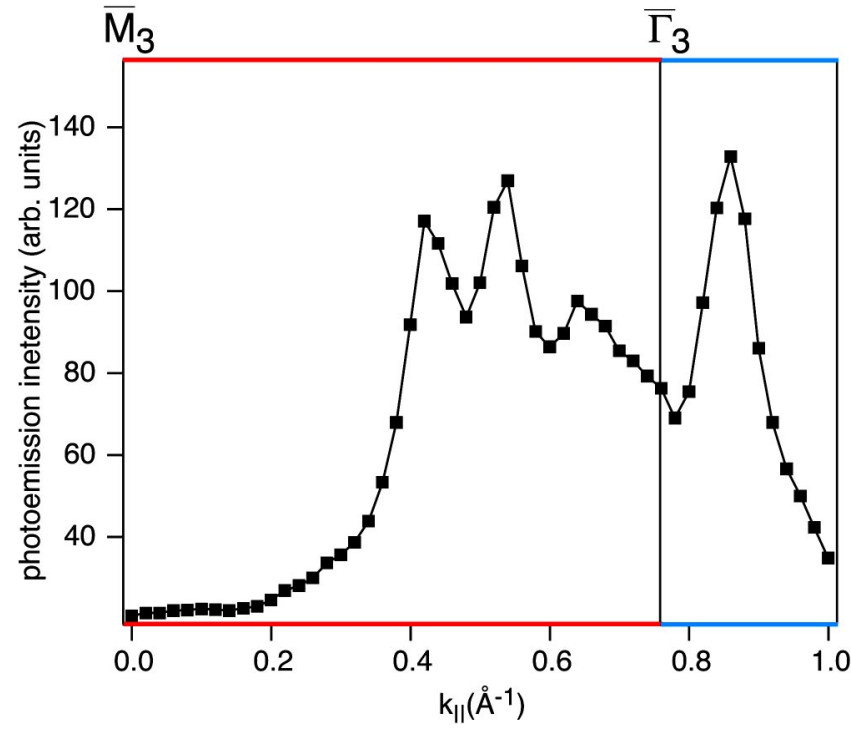

FIG. 4. (Color online) Integrated photoemission from Fig. 3 along $\bar{\Gamma}_{3}-\bar{M}_{3}$, integrated over a $20-\mathrm{meV}$-wide window centered at the Fermi energy. The maxima in this curve correspond approximately to the positions of the Fermi-level crossings.

20-meV-wide window centered on the Fermi level. This shows all three crossings, as well as the crossing in the $\bar{\Gamma}_{3}-\bar{K}_{3}$ direction, as sharp peaks.

The only other clear feature near the Fermi level is found near the $\bar{M}^{\prime}$ points. The Fermi contour of this state is not resolved in the experiment, most probably because of the very close proximity of the crossings. This leaves us with a surface Fermi surface containing three segments: an electron pocket around $\bar{\Gamma}$, the two (equivalent) hole pockets centered on the $\bar{\Gamma}-\bar{M}$ lines of the SBZ, and the Fermi-surface segment near $\bar{M}^{\prime}$. The data taken around the other two $\bar{\Gamma}$ points appear to be consistent with this picture, even though only the $\bar{\Gamma}_{3}-\bar{M}_{3}$ direction shows all three crossings very clearly. Figure 5 shows the result of a Fermi-surface mapping in a irreducible part of the SBZ, including the $\bar{\Gamma}_{3}$ point. Shown is the photoemission intensity integrated over a $31 \mathrm{meV}$ window centered on the Fermi level minus the photoemission intensity in a window of the same size, but $70 \mathrm{meV}$ above the Fermi level. The three Fermi-surface elements are easily recognized. The electron pocket around $\bar{\Gamma}_{3}$ has a slightly elliptical shape, and the hole pocket is approximately circular. The Fermi surface segment near $\bar{M}^{\prime}$ appears as a stripe. A closer inspection of the figure reveals that the $\bar{\Gamma}_{3}$ point appears to be slightly shifted from its expected position. This is probably an artifact caused by the rapid change in the photoemission intensity when going to high off-normal emission angles. Our figure shows the raw data; i.e., no correction of this effect is applied. Apart from the three "true" Fermisurface features there are also other structures, in particular the high intensity along the $\bar{\Gamma}_{3}-\bar{M}_{3}$ and close to $\bar{K}_{2}$. These can also be found at the corresponding regions of the extendedzone scheme in a larger scan. The high intensity close to $\bar{K}_{2}$ is clearly related to the surface state at this point, which is 


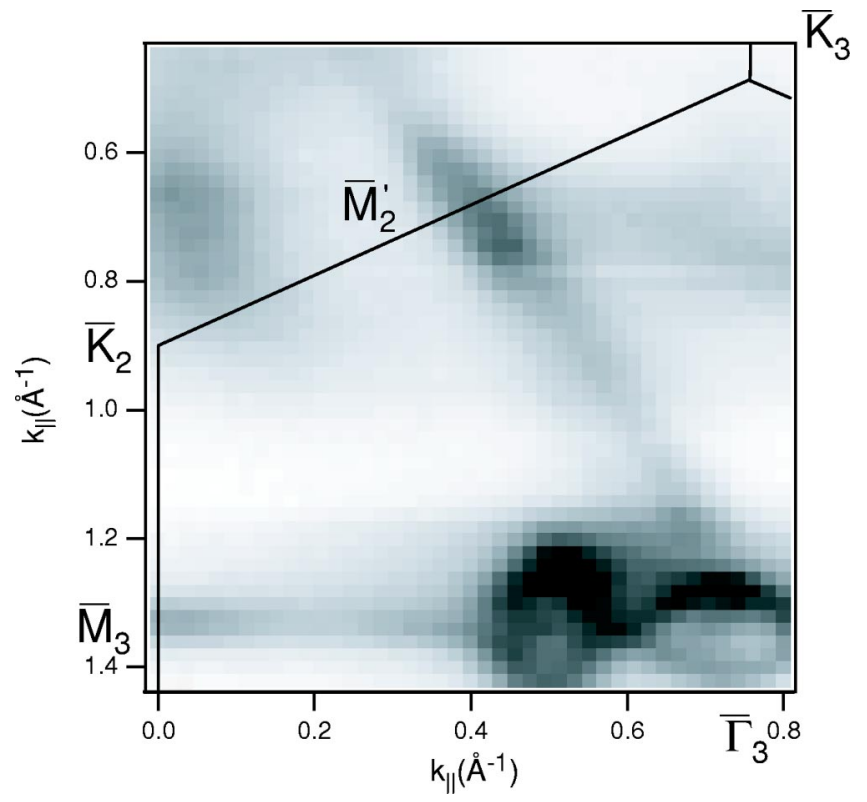

FIG. 5. Normalized photoemission intensity at the Fermi level (see text). Black corresponds to high intensity. The solid line is the SBZ boundary.

very close to the Fermi level, close enough to increase the photoemission intensity at the Fermi edge by its finite width, but it does not cross it (see Fig. 3). The other structure is related to the bulk states. The width of the projected bulk band gap along $\bar{\Gamma}-\bar{M}$ is relatively low (as can be seen in Fig. 3 ). This means that a broad bulk peak is relatively close to the Fermi level in a large range of $\vec{k}_{\|}$, and what we see in the Fermi-surface map is the remaining intensity at the wings of this peak. In Fig. 3 the intensity at $\bar{M}_{3}$ appears to be very weak and the bulk feature is invisible, but this is just a result of the scaling. A scan perpendicular $\bar{\Gamma}_{3}-\bar{M}_{3}$ line (not shown) confirms the picture just given.

From the data of Fig. 3 and the energy-distribution curves (EDCs) on which the construction of Fig. 3 was based, we can make a reasonable estimate of the Fermi velocity of the surface states forming the surface-Fermi contour. We obtain values of $1.5 \mathrm{eV} \AA, 3.0 \mathrm{eV} \AA$, and $4.9 \mathrm{eV} \AA$ for the hole pocket along $\bar{\Gamma}_{3}-\bar{M}_{3}$, the electron pocket along $\bar{\Gamma}_{3}-\bar{M}_{3}$, and the electron pocket along $\bar{\Gamma}_{3}-\bar{K}_{3}$, respectively. Thus, the bands forming the Fermi surface are rather steep, but not as the steep as the bands forming the bulk Fermi surface, where the Fermi velocity can be up to $20 \mathrm{eV} \AA$.

In a previous work $^{21}$ we pointed out the importance of spin-orbit coupling effects in the interpretation of the electronic structure of the Bi surfaces. A very large, Rashba-like splitting of the surface states and the steepness of the bands at the Fermi level make it often very difficult to clearly identify the spin-split partners of the bands from the occupied part of the band structure only. Therefore, we made use of $a b$ initio calculations of these surfaces to get a clearer picture of the surface electronic structure.

In Fig. 6 we show the result of an ab initio band structure calculation of the relaxed $\mathrm{Bi}(100)$ surface. Around the Fermi level, we find a band of surface states extending through the

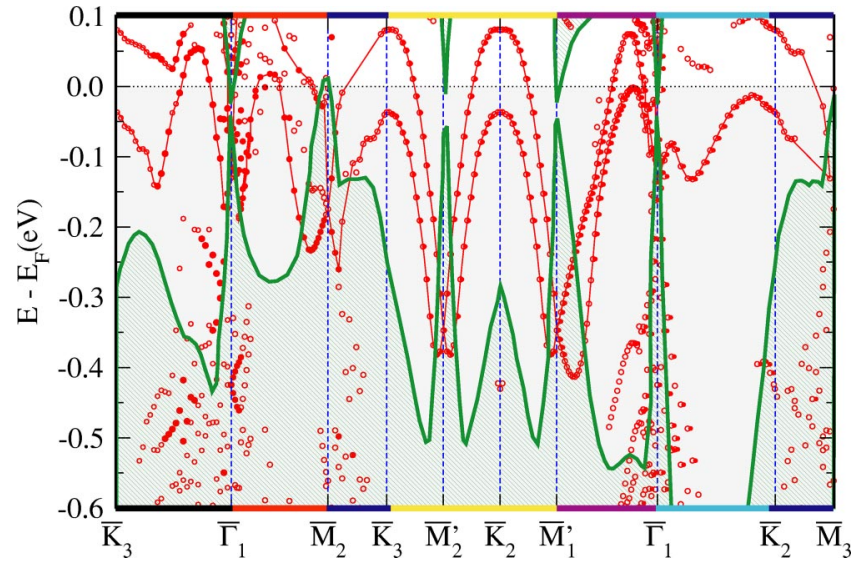

FIG. 6. (Color online) Band structure of a 22-layer Bi(100) film terminated with $\mathrm{H}$ on the lower side. The states originating from the upper (clean) half of the film are marked red, while states from the lower half are not displayed. The states with a large weight near the surface are marked as filled red circles. The projected bulk bands are shown as a green hashed area. A tentative connection between the surface states is marked by a red line. The colored horizontal bars can be used for a direct comparison with the equivalent directions in Fig. 3.

whole surface Brillouin zone. Spin-orbit coupling splits this band into two branches, at the $\bar{\Gamma}, \bar{M}^{\prime}$, and $\bar{M}$ points that these branches have to cross. ${ }^{21}$ At the $\bar{M}_{2}^{\prime}$ and $\bar{M}_{1}^{\prime}$ points, a Rashbalike signature of two parabolic bands shifted in positive and negative directions in momentum space is clearly visible. In between, one band crosses the Fermi level, while the other one remains completely occupied. The same situation can be found in the $\bar{M}_{1}^{\prime}-\bar{\Gamma}_{1}$ direction, while in the $\bar{\Gamma}_{1}-\bar{K}_{2}$ direction the upper branch is almost completely unoccupied and crosses the Fermi level once more close to $\bar{M}_{3}$. This scenario is very similar to that found on the $\mathrm{Bi}(111)$ surface, where all the $\bar{M}$ as well as the $\bar{K}$ points are equivalent. ${ }^{21}$ The situation is much more involved in the $\bar{K}_{3}-\bar{\Gamma}_{1}-\bar{M}_{2}-\bar{K}_{3}$ section of the band structure, where the projected bulk states are closer to the Fermi level. To facilitate discussion, a tentative connection between the surface states is marked by a red line in Fig. 6.

Comparing the band structure of Fig. 6 with the measured dispersion (Fig. 3), in most parts the connection between theory and experiment can now be established. The regions of high photoemission intensity around $\bar{\Gamma}_{1}$ also show a high density of surface states or near-surface states in the calculation. The three Fermi-level crossings near the Brillouin zone center in the $\bar{\Gamma}_{1}-\bar{M}_{2}$ direction are visible, as well as the fact that there are only two such crossings in $\bar{\Gamma}_{1}-\bar{M}_{1}^{\prime}$ direction. Nevertheless, some differences between theory and experiment can be realized. The minimum of the band in $\bar{\Gamma}_{1}-\bar{K}_{2}$ direction is found at higher binding energies in experiment than in theory and the "shoulder" observed in the equivalent $\bar{\Gamma}_{3}-\bar{K}_{3}$ cannot be found in the calculations. At first glance, the situation near the $\bar{M}^{\prime}$ points also appears to be quite different in theory and experiment. The experiment re- 


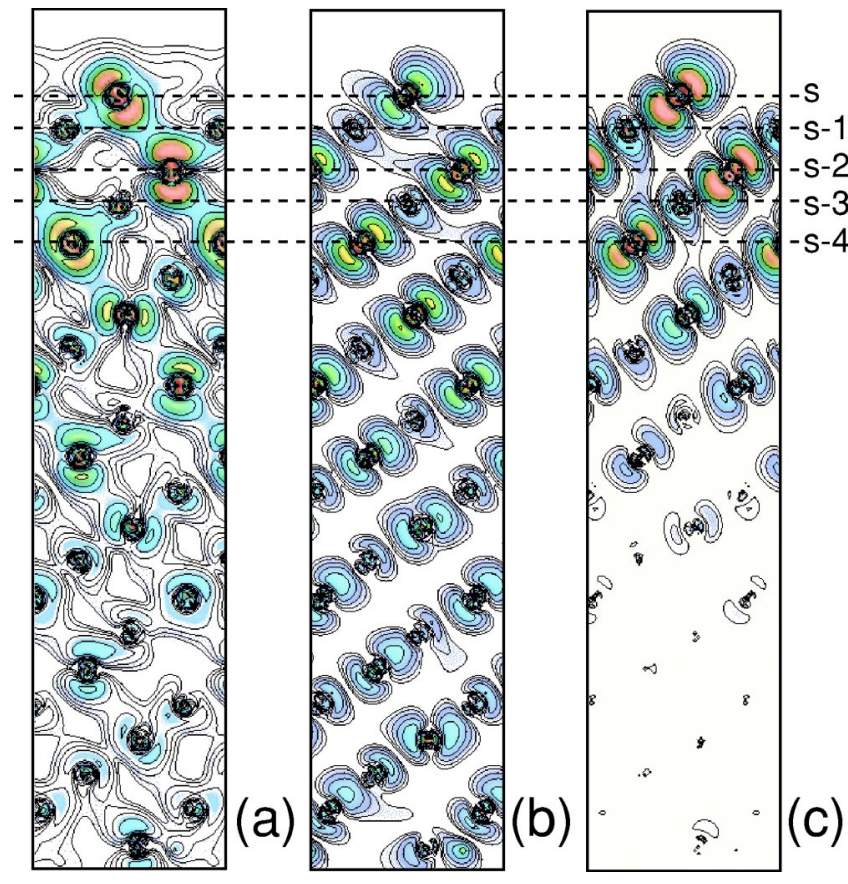

FIG. 7. (Color online) Charge-density contours of surface states on the $\operatorname{Bi}(100)$ surface: (a) is a state from the middle of $\bar{\Gamma}_{1}-\bar{M}_{2}$ almost at the Fermi level, (b) is the highest-occupied state at $\bar{K}_{2}$, and (c) is a state from the middle of the highest-occupied band in the $\bar{\Gamma}_{1}-\bar{K}_{2}$ direction. The position of the surface plane $(s)$ and of the four lower layers are indicated by the dashed lines. The contour lines are plotted on a logarithmic scale.

veals a unclear feature at the Fermi level exactly at $\bar{M}^{\prime}$, whereas the calculation shows clearly separated Fermi-level crossings of the surface state around $\bar{M}^{\prime}$. However, this apparent discrepancy is not a severe one, because a small upward shift of the surface-state energy at $\bar{M}^{\prime}$ in the calculation would lead to Fermi-level crossings much closer to $\bar{M}^{\prime}$ and in better agreement with the experiment. Note that the dispersion of the surface states around $\bar{M}^{\prime}$ is slightly different from the result shown in Ref. 21. This is caused by the relaxation of the surface geometry.

On the whole, the comparison between experiment and theory for $\mathrm{Bi}(100)$ is less favorable than for $\mathrm{Bi}(111)$ (Ref. 22) and $\mathrm{Bi}(110),{ }^{23}$ for which almost quantitative agreement can be achieved. This is also the reason why we have chosen not to plot the experimental dispersion on the same graph as the theoretical one. The main cause for the limited accuracy of the calculation for $\mathrm{Bi}(100)$ can be understood by inspecting the charge-density contours of different surface states shown in Fig. 7. Some states near the Fermi level penetrate very deeply into the bulk [Figs. 7(a) and 7(b)], while others [Fig. $7(\mathrm{c})$ ] are localized in the first five layers. This penetration depth is remarkably high, compared to $\mathrm{Bi}(111)$ or $\mathrm{Bi}(110)$, in which the surface states are mostly localized in the first bilayer of the surface. ${ }^{22}$ Also the weight of these states in the vacuum is rather low. In some cases, e.g., at the highest occupied state at the $\bar{K}_{2}$ point, the state even has its largest weight in the fifth layer from the surface [cf. Fig. 7(b)], even though it is clearly in a large projected-bulk band gap. This limits the possible accuracy of the theory, which is restricted to a film of finite thickness.

The strong penetration of the surface states on $\mathrm{Bi}(100)$ could be important for understanding some results concerning the electronic structure of $\mathrm{Bi}$ clusters and nanotubes. Weitzel and Micklitz reported the observation of superconductivity in granular films of Bi clusters. ${ }^{6}$ The structure of the clusters was rhombohedral, as in bulk Bi, but bulk $\mathrm{Bi}$ is not superconducting. An initial interpretation of this finding was the occurrence of surface superconductivity on the clusters. This appeared to be consistent with a number of experimental findings: a linear increase of $T_{C}$ for a smaller clusters of size $L$ and a dependence of $T_{C}$ on the chemical nature of the embedding matrix. Later, the view of a two-component cluster (metallic surface and semimetallic bulk) was challenged by the same group. ${ }^{24}$ The normal and superconducting properties showed a very similar dependence on the cluster size, suggesting that dividing the system into two independent components might be inappropriate. Deeply penetrating surface states could well be the cause for this apparent contradiction. States similar to those in Figs. 7(a) and 7(b) could exist on the (100) face of the clusters. Strictly spoken, the states are surface states and therefore influenced by, e.g., the surroundings of the crystal. At the same time, they would be delocalized over a cluster that is a few nanometers in size. Similar surface states could inhibit predicted ${ }^{8}$ semimetal-to-semiconductor transitions in Bi nanowires. ${ }^{9}$

With the use of Fig. 6, one can try to construct the Fermi surface from the calculated band structure and compare it with the measured plot in Fig. 5. A proper construction of the theoretical Fermi surface would require calculations of the dispersion along many directions, not only those shown in Fig. 6. This would be quite expensive from a computational point of view, and it would not lead to more physical insight because of the limited accuracy discussed above. Around both the $\bar{\Gamma}$ and the $\bar{M}$ points we identify small electron pockets, while along the $\bar{\Gamma}-\bar{M}$ line a hole pocket is formed. Since

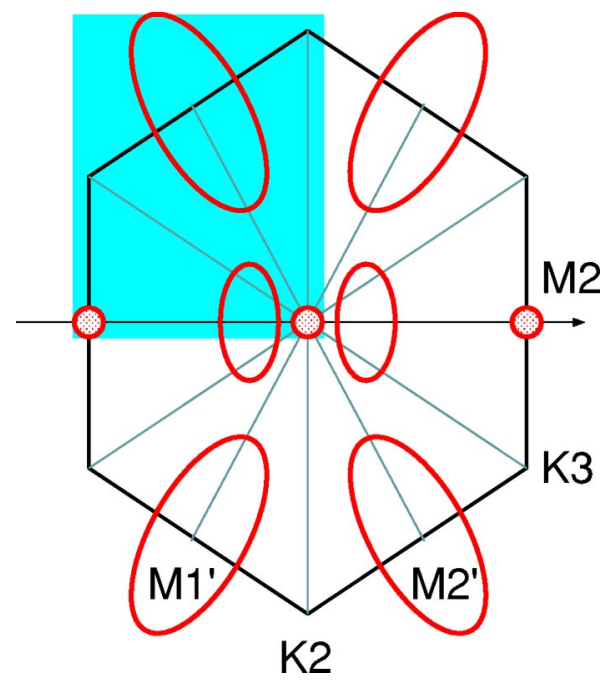

FIG. 8. (Color online) Sketch of the Fermi surface as deduced from the band structure in Fig. 6. The turquoise rectangle marks the part plotted in Fig. 5. 
a similar feature is also found along the $\bar{\Gamma}_{1}-\bar{K}_{3}$ line, we conclude that this pocket is rather elliptical, as indicated in the sketch in Fig. 8. (Of course we cannot exclude the possibility that these two features are not connected and belong to two separate pockets.) These findings compare well with the experimental Fermi surface shown in Fig. 5. Near $\bar{M}^{\prime}$ the theory predicts very large Fermi-surface features in clear disagreement with the experiments that show a broad feature at $\bar{M}^{\prime}$, without resolving any details. As already discussed above, this apparent discrepancy is probably caused by a small error in the calculated binding energy of the surface state that has a considerable impact on the size of the related Fermi-surface element. It is interesting to point out that the Fermi surface of $\mathrm{Bi}(100)$ bears some similarity to the Fermi surface of $\operatorname{Bi}(111) .{ }^{12,25}$ In particular the situation near $\bar{\Gamma}$ in the direction of the mirror line is very similar.

\section{CONCLUSIONS}

We have presented a combined experimental and theoretical investigation of the electronic structure of $\mathrm{Bi}(100)$. As with the $\mathrm{Bi}(110)$ and (111) surfaces, the $\mathrm{Bi}(100)$ surface shows several surface-state-related Fermi-level crossings.
The surface Fermi surface shows some similarities to that of the $\mathrm{Bi}(111)$ surface, which is similar from a geometrical point of view, but the character of the surface states is quite different. Whereas all the states are fairly surface localized on $\operatorname{Bi}(111)$ and $\operatorname{Bi}(110)$, they can penetrate deeply into the crystal on $\mathrm{Bi}(100)$. This could have profound implications for the electronic structure of Bi clusters and nanowires, but it also limits the accuracy of calculations (e.g., of the Fermi energy) when a film of finite thickness is used to simulate the semi-infinite crystal. Nevertheless, reasonable agreement is found between our experimental results and calculations. The calculations also show that the surface-state bands are split by spin-orbit interaction in the whole surface Brillouin zone, apart from some high-symmetry points. Finally, the strong $\vec{k}_{\|}$ dependence of the surface-state penetration can also be expected to contribute to the variations of the electron-phonon coupling strength observed on $\mathrm{Bi}(100),{ }^{26}$ because it causes the surface-state electrons to interact with different types of phonons for different $\vec{k}_{\|}$points.

\section{ACKNOWLEDGMENT}

This paper was supported by the Danish National Science Foundation.
*Electronic address: philip@phys.au.dk

${ }^{1}$ V. S. Édel'man, Adv. Phys. 25, 555 (1976).

${ }^{2}$ J.-P. Issi, Aust. J. Phys. 32, 585 (1979).

${ }^{3}$ V. N. Lutskii, Sov. Phys. JETP 2, 1245 (1965).

${ }^{4}$ V. B. Sandomirskii, Sov. Phys. JETP 25, 101 (1967).

${ }^{5}$ Y. F. Ogrin, V. N. Lutskii, and M. I. Elinson, JETP Lett. 3, 71 (1966).

${ }^{6}$ B. Weitzel and H. Micklitz, Phys. Rev. Lett. 66, 385 (1991).

${ }^{7}$ L. D. Hicks and M. S. Dresselhaus, Phys. Rev. B 47, R16 631 (1993).

${ }^{8}$ Y.-M. Lin, X. Sun, and M. S. Dresselhaus, Phys. Rev. B 62, 4610 (2000)

${ }^{9}$ T. E. Huber, A. Nikolaeva, D. Gitsu, L. Konopko, C. A. Foss Jr., and M. J. Graf, Appl. Phys. Lett. 84, 1326 (2004).

${ }^{10}$ S. Agergaard, C. Søndergaard, H. Li, M. B. Nielsen, S. V. Hoffmann, Z. Li and Ph. Hofmann, New J. Phys. 3, 15.1 (2001).

${ }^{11}$ M. Hengsberger, P. Segovia, M. Garnier, D. Purdie, and Y. Baer, Eur. Phys. J. B 17, 603 (2000).

${ }^{12}$ C. R. Ast and H. Höchst, Phys. Rev. Lett. 87, 177602 (2001).

${ }^{13}$ F. Jona, Surf. Sci. 8, 57 (1967).

${ }^{14}$ C. Søndergaard, Ph.D. thesis, University of Aarhus, 2001.

${ }^{15}$ S. V. Hoffmann, C. Søndergaard, C. Schultz, Z. Li and Ph. Hofmann, Nucl. Instrum. Methods Phys. Res. A 523, 441 (2004).
${ }^{16}$ H. Krakauer, M. Posternak, and A. J. Freeman, Phys. Rev. B 19, 1706 (1979).

${ }^{17}$ E. Wimmer, H. Krakauer, M. Weinert, and A. J. Freeman, Phys. Rev. B 24, 864 (1981).

${ }^{18}$ V. L. Moruzzi, J. F. Janak, and A. R. Williams, Calculated Electronic Properties of Metals (Pergamon, New York, 1978).

${ }^{19}$ C. Li, A. J. Freeman, H. J. F. Jansen, and C. L. Fu, Phys. Rev. B 42, 5433 (1990).

${ }^{20}$ Y. Liu and R. E. Allen, Phys. Rev. B 52, 1566 (1995).

${ }^{21}$ Y. M. Koroteev, G. Bihlmayer, J. E. Gayone, E. V. Chulkov, S. Blügel, P. M. Echenique and Ph. Hofmann, Phys. Rev. Lett. 93, 046403 (2004).

${ }^{22}$ Y. M. Koroteev, G. Bihlmayer, and E. V. Chulkov (unpublished).

${ }^{23}$ J. I. Pascual, G. Bihlmayer, Y. M. Koroteev, H.-P. Rust, G. Ceballos, M. Hansmann, K. Horn, E. V. Chulkov, S. Blügel, P. M. Echenique, and Ph. Hofmann, Phys. Rev. Lett. 93, 196802 (2004).

${ }^{24}$ C. Vossloh, M. Holdenried, and H. Micklitz, Phys. Rev. B 58, 12422 (1998).

${ }^{25}$ C. R. Ast and H. Höchst, Phys. Rev. B 67, 113102 (2003).

${ }^{26}$ J. E. Gayone, S. V. Hoffmann, Z. Li, and Ph. Hofmann, Phys. Rev. Lett. 91, 127601 (2003). 